\title{
An Improved Multicell MMSE Channel Estimation in a Massive MIMO System
}

\author{
Ke Li, ${ }^{1}$ Xiaoqin Song, ${ }^{2}$ M. Omair Ahmad, ${ }^{3}$ and M. N. S. Swamy ${ }^{3}$ \\ ${ }^{1}$ College of Information Technology, Beijing Union University, Beijing 100010, China \\ ${ }^{2}$ College of Electronics and Information Engineering, Nanjing University of Aeronautics and Astronautics, Nanjing 210016, China \\ ${ }^{3}$ Department of Electrical and Computer Engineering, Concordia University, Montreal, QC, Canada H3G IM8
}

Correspondence should be addressed to Ke Li; like@buu.edu.cn

Received 22 January 2014; Accepted 9 April 2014; Published 8 May 2014

Academic Editor: Kan Zheng

Copyright (C) $2014 \mathrm{Ke} \mathrm{Li} \mathrm{et} \mathrm{al.} \mathrm{This} \mathrm{is} \mathrm{an} \mathrm{open} \mathrm{access} \mathrm{article} \mathrm{distributed} \mathrm{under} \mathrm{the} \mathrm{Creative} \mathrm{Commons} \mathrm{Attribution} \mathrm{License,} \mathrm{which}$ permits unrestricted use, distribution, and reproduction in any medium, provided the original work is properly cited.

\begin{abstract}
Massive MIMO is a promising technology to improve both the spectrum efficiency and the energy efficiency. The key problem that impacts the throughput of a massive MIMO system is the pilot contamination due to the nonorthogonality of the pilot sequences in different cells. Conventional channel estimation schemes cannot mitigate this problem effectively, and the computational complexity is increasingly becoming larger in views of the large number of antennas employed in a massive MIMO system. Furthermore, the channel estimation is always carried out with some ideal assumptions such as the complete knowledge of largescale fading. In this paper, a new channel estimation scheme is proposed by utilizing interference cancellation and joint processing. Highly interfering users in neighboring cells are identified based on the estimation of large-scale fading and then included in the joint channel processing; this achieves a compromise between the effectiveness and efficiency of the channel estimation at a reasonable computational cost, and leads to an improvement in the overall system performance. Simulation results are provided to demonstrate the effectiveness of the proposed scheme.
\end{abstract}

\section{Introduction}

Recently, there has been a great deal of interests in MIMO systems using very large antenna arrays, namely, the massive MIMO or full dimension MIMO (FD-MIMO) systems [1-4]. Such systems can provide a significant increase in reliability, data rate, and energy efficiency with simple signal processing [1]. When the number of base station (BS) antennas grows large, the channel vectors between the users and the BS become very large-size random vectors and under "favorable propagation" conditions. Then, they become pairwise orthogonal. As a consequence, with simple linear processing, for example, maximum-ratio combining (MRC), or zero forcing (ZF), assuming that the BS has perfect channel state information (CSI), the interference from other users can be cancelled without consuming more time-frequency resources. This dramatically increases the spectral efficiency. Furthermore, by using a very large antenna array at BS, the transmit power can be drastically reduced.
Practically, however, the BS does not have perfect CSI. Instead, it estimates the channels as usual. Conventionally it is done by using uplink pilots. Since both the timefrequency resources allocated for pilot transmission and the channel coherence time are limited, the number of possible orthogonal pilot sequences is limited too, and hence, the pilot sequences have to be reused in neighbouring cells of cellular systems. Therefore, channel estimates obtained in a given cell get contaminated by the pilots transmitted by the users in other cells. This causes pilot contamination [5]; that is, the channel estimate at the base station in one cell becomes polluted by the pilots of the users from other cells.

Considering the importance of pilot contamination mitigation to guarantee the good performance of massive MIMO, many schemes, ranging from optimal pilot design and allocation, advanced channel estimation, to downlink precoding [5-11], have been proposed to deal with this problem.

In [10], a pilot design criterion and Chu sequence-based pilots have been proposed by exploiting the orthogonality 
of the channel vectors of massive MIMO systems. Because of the proposed pilots, the channel estimate of most terminals of a cell is only interfered by partial cells rather than all the other cells, where the latter is caused by traditional pilots. As a result, pilot contamination is mitigated. It is also possible to coordinate the use of pilots or adaptively allocate the pilot sequences to different terminals in the network [8].

Multicell joint channel estimation algorithms $[5,8]$ or even blind techniques that circumvent the use of pilots altogether [7] may mitigate or eliminate the effects of pilot contamination. The approach in [7] exploits the asymptotic orthogonality of the channel vectors in very large MIMO systems and shows that the channel to each user can be estimated from the covariance matrix of the received signals, up to a remaining scalar multiplicative ambiguity, which can be resolved by utilizing a short training sequence.

The new precoding techniques, such as pilot contamination precoding [11], that take into account the network structure can utilize cooperative transmission over a multiplicity of cells-outside of the beamforming operation-to nullify, at least partially, the directed interference that results from pilot contamination. Unlike coordinated beamforming over multiple cells which requires estimates of the actual channels between the terminals and the service-arrays of the contaminating cells, the pilot-contamination precoding requires only the corresponding slow-fading coefficients.

By overviewing the abovementioned channel estimation schemes, it can be seen that, for simplicity, it is always assumed that the large-scale fading propagation (i.e., pathloss and shadow fading) is fixed and known a priori, which is not the case in practice. Furthermore, the computational complexity of conventional channel estimations such as least square (LS) and minimum mean square error (MMSE) estimations, grows sharply in a massive MIMO system, as the number of antennas is much more than that in the current multiple antennas systems. This problem is getting worse in the case of joint processing in a multicell scenario. As a countermeasure to these problems, an improved channel estimation scheme, which aims at mitigating the pilot contamination at an affordable expense and in a more realistic situation, is proposed in this paper.

To deal with the abovementioned problems, a new channel estimation scheme is proposed by utilizing interference cancellation and joint processing. Highly interfering users in neighboring cells are identified based on the estimation of large-scale fading and then included in the joint channel processing; this achieves a compromise between the effectiveness and efficiency of the channel estimation and leads to an improvement in the overall system performance. Metrics of the performance evaluation of the channel estimation are defined, and finally simulation results are provided which validate the proposed scheme.

The paper is organized as follows. Section 2 gives the multicell massive MIMO system model and the pilot design. The conventional channel estimator and the proposed multicell joint MMSE channel estimation are presented in Section 3. Section 4 presents the simulation results and the analysis of computational complexity of the proposed scheme. Finally, Section 5 concludes this paper.
Notations. $(\cdot)^{*},(\cdot)^{T}$, and $(\cdot)^{H}$ denote the conjugate, transpose, and conjugate transpose, respectively, $[\cdot]_{i, j}$ is the $(i, j)$ th entry of a matrix, $E\{\cdot\}$ is the expectation, and $\|\cdot\|$ denotes the Frobenius norm.

\section{Multicell Massive MIMO System Model}

2.1. System Model. Here we consider a massive MIMO cellular system with $L$ hexagonal cells, as depicted in Figure 1. Each cell comprises one base station with $M$ antennas and $K$ single antenna users $(K \ll M$, in the case of a massive MIMO system). All the cells share the same band of frequencies. In addition, the system employs OFDM and works in timedivision duplex (TDD) mode.

Let the average power (during transmission) at the base station be $p_{f}$ and let the average power at each user be $p_{r}$. In one coherence interval, the channel responses of $N_{a}$ symbols are assumed to be static, which means that the channel estimate is effective only in this interval. Consequently, a total of $N_{c}$ symbols are used for pilots from all the terminals to BS, and the remaining $N_{a}-N_{c}$ symbols are used for a two-way data transmission. Apart from that, following the model in [1], the channel responses are assumed to be constant in the frequency smooth interval, which consists of $N_{s}$ consecutive subcarriers. Therefore, to estimate the channel response of one subcarrier, every terminal can transmit pilots over $N_{s}$ subcarriers. In the following, channel responses of the $N_{s}$ subcarriers will be estimated. As a result, the pilot length is given by $\tau=N_{c} N_{s}$, and thus up to $\tau$ terminals can transmit pilots to BS without intracell interference.

The propagation factor (channel response) between the $m$ th base station antenna of the $l$ th cell and the $k$ th user of the $j$ th cell is given by

$$
g_{j l k m}=\sqrt{\beta_{j l k}} h_{j l k m},
$$

where $\left\{\beta_{j l k}\right\}$ are the large-scale fading coefficients, composed of pathloss and shadow fading, and are assumed invariant for different antennas of the same BS, and $\left\{h_{j l k m}\right\}$ are the smallscale fading coefficients and are assumed to be independent and identically distributed (i.i.d.) and circularly symmetric Gaussian random variables $(\mathscr{C} \mathscr{N}(0,1))$. We assume the channel reciprocity for the downlink and the uplink, that is, the propagation factor $h_{j l k m}$ is the same for both the downlink and the uplink, and block fading, that is, $\left\{h_{j l k m}\right\}$ remains constant for the duration of coherence intervals (typically 430 OFDM symbols). The additive noise signals at all terminals are i.i.d. $(\mathscr{C} \mathcal{N}(0,1))$ random variables. Then, the channel matrix from the terminals of the $j$ th cell to BS in the $l$ th cell is given by

$$
\mathbf{G}_{j l}=\mathbf{D}_{j l} \mathbf{H}_{j l} \in \mathbb{C}^{K \times M},
$$

where $\mathbf{H}_{j l} \in \mathbb{C}^{K \times M}$ are small-scale fading coefficients, that is, $\left[\mathbf{H}_{j l}\right]_{k, m}=h_{j l k m} ; \mathbf{D}_{j l} \in \mathbb{R}^{K \times K}$ is a diagonal matrix, where $\left[\mathbf{D}_{j l}\right]_{k, k}=\sqrt{\beta_{j l k}}$.

At the beginning of every coherence interval, all the users in all the cells synchronously transmit pilot sequences of 


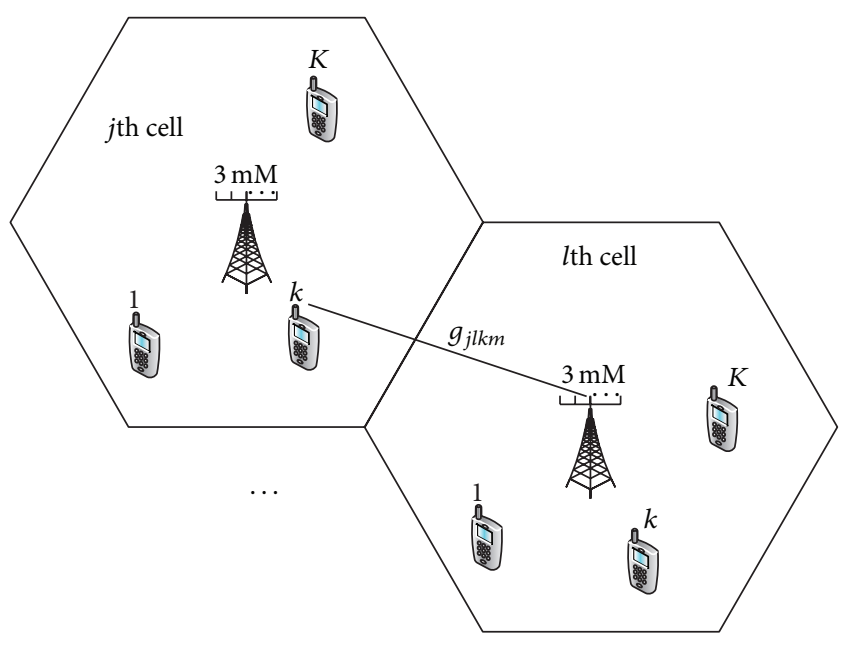

FIGURE 1: A multicell massive MIMO system model.

length of $\tau$ symbols. Let $\sqrt{\tau} \underline{\psi}_{j k}$ (where $\left.\underline{\psi}_{j k}^{H} \underline{\psi}_{j k}=1\right)$ be the pilot vector transmitted by the $k$ th user in the $j$ th cell and consider the base station of the $l$ th cell. Then, the vector received at the $m$ th antenna is given by

$$
\underline{y}_{l m}=\sum_{j=1}^{L} \sum_{k=1}^{K} \sqrt{p_{r} \tau \beta_{j l k}} h_{j l k m} \underline{\psi}_{j k}+\underline{w}_{l m},
$$

where $\underline{w}_{l m} \in \mathbb{C}^{\tau \times 1}$ is the additive noise. Let $\mathbf{Y}_{l}=\left[\begin{array}{llll}y_{l 1} & \underline{y}_{l 2} & \cdots & \underline{y}_{l M}\end{array}\right] \in \mathbb{C}^{\tau \times M}, \mathbf{W}_{l}=$ $\left[\begin{array}{llll}\underline{w}_{l 1} & \underline{w}_{l 2} & \cdots & \underline{w}_{l M}\end{array}\right] \in \mathbb{C}^{\underline{\tau \times M}}, \Psi_{j}, j=1, \ldots L$, be the system matrices and $\Psi_{j}=\left[\begin{array}{llll}\underline{\psi}_{j 1} & \underline{\psi}_{j 2} & \cdots & \underline{\psi}_{j K}\end{array}\right] \in \mathbb{C}^{\tau \times K}$. From (1), the signal received at the $l$ th base station is given by

$$
\mathbf{Y}_{l}=\sqrt{p_{r} \tau} \sum_{j=1}^{L} \Psi_{j} \mathbf{G}_{j l}+\mathbf{W}_{l} .
$$

By separating the received pilots of the users in the target cell and that in the neighboring cells, (4) can be rewritten as

$$
\mathbf{Y}_{l}=\sqrt{p_{r} \tau} \Psi_{l} \mathbf{G}_{l l}+\sqrt{p_{r} \tau} \sum_{j=1, j \neq l}^{L} \Psi_{j} \mathbf{G}_{j l}+\mathbf{W}_{l}
$$

in which the first item is the desired received pilots, and the second one is the pilots from neighboring cells, that is, the interfering pilot signals.

2.2. Pilot Design. It is known that the number of pilot symbols is limited by the channel coherence interval; thus, it is assumed that $\tau<2 K$. In addition, the system matrix $\Psi_{l}$ must satisfy the requirement $\boldsymbol{\Theta}_{l l}=I_{K}$, where $\boldsymbol{\Theta}_{j l}=\boldsymbol{\Psi}_{l}^{T} \boldsymbol{\Psi}_{j}^{*} \in \mathbb{C}^{K \times K}$ is the correlation matrix between the pilots. Consequently, $\tau \geq K$ is required, which means that the number of users per cell $K$ should not be larger than the pilot length $\tau$. Thus, the pilot length should lie in the interval $K \leq \tau<2 K$.

In [10], a pilot design method has been proposed based on the Chu sequences, which have perfect autocorrelation property and very low cross correlations. The pilots inside a cell are orthogonal because of different cyclic shifts. These pilots are reused among the $L$ cells after being multiplied by phase shifts. Then, the cross-correlation values of the pilots are different for different terminals, which fit the scenarios with large shadow fading variance. Moreover, it is proved that the channel estimate of most terminals is only interfered by partial cells. As a result, the pilot contamination is mitigated. We will now briefly describe how the pilots are generated.

The cell-specific basic pilot vector of users in the $j$ th cell is denoted as $\sqrt{\tau} \underline{\psi}_{j}$, and the $n$th entry of $\underline{\psi}_{j}$ is given by

$$
\left[\underline{\psi}_{j}\right]_{n}=a_{n} \exp i \frac{2 \pi q_{j} n}{\tau}, \quad n=1,2, \ldots, \tau,
$$

where $\exp i\left(2 \pi q_{j} n / \tau\right)$ is the phase shift between the cells, $q_{j}$ is an integer that is not exactly divisible by $\tau$ and differs from $j$, and $a_{n}$ is the $n$th entry of the Chu sequence $\mathbf{a}=\left[a_{0}\right.$, $\left.a_{1}, \ldots, a_{\tau-1}\right]$ and can be expressed as [12]

$$
a_{n}=\exp i \frac{N \pi}{\tau} n(n+(\tau \bmod 2)), \quad n=0,1, \ldots, \tau-1
$$

$N$ is the unique sequence parameter, which should be an integer that is relatively prime to $\tau$. The pilot vectors of users in one cell are the cyclic shifted versions of the cell-specific basic pilot vector. The pilot vector of the $k$ th user in the $j$ th cell is given by

$$
\sqrt{\tau} \underline{\psi}_{j k}=\left\langle\sqrt{\tau} \underline{\psi}_{j}\right\rangle_{k-1}
$$

in which $\langle\cdot\rangle_{k}$ is the left circular shift of a vector with shift length $k$.

The circular autocorrelation function of the Chu sequence $\mathbf{a}$ is defined as

$$
r_{j}=\sum_{n=0}^{\tau-1} a_{n} a_{(n+j) \bmod \tau}^{*}, \quad j=0,1, \ldots, \tau-1 .
$$

Then, the autocorrelation values are $r_{0}=\tau, r_{j}=0$ when $j \neq 0$. It can be easily seen that the autocorrelations of vectors $\underline{\psi}_{j k}$ have the same property.

\section{Multicell MMSE Channel Estimation}

3.1. Conventional Single-Cell Channel Estimation. Conventional channel estimation relies on correlating the received signal with the known pilot sequence. The typical one is referred here as the LS estimation. For the abovementioned system model, the LS estimator for the desired channel of the target cell $l$ is given by [8]

$$
\widehat{\mathbf{H}}_{l l}=\sqrt{p_{r} \tau}\left(\mathbf{D}_{l l} \boldsymbol{\Psi}_{l}^{H} \boldsymbol{\Psi}_{l} \mathbf{D}_{l l}\right)^{-1} \mathbf{D}_{l l} \boldsymbol{\Psi}_{l}^{H} \mathbf{Y}_{l}
$$

The conventional estimator suffers severely from a lack of orthogonality between the desired pilots and the interfering ones. In particular, when the same pilot sequence is reused in all the $L$ cells, the estimator can be written as

$$
\widehat{\mathbf{H}}_{l l}=\mathbf{H}_{l l}+\sum_{j=1, j \neq l}^{L} \mathbf{H}_{j l}+\frac{\boldsymbol{\Psi}_{l}^{H} \mathbf{W}_{l}}{\tau}
$$


which shows that the interfering channels leak directly into the desired channel estimate.

Another well-known linear channel estimation is the MMSE estimator, which is known to have a better performance. For the pilot signal received at the $l$ th base station as described in (4), if we treat the pilots of other cells arriving at the antennas of $l$ th BS as pure interference, then the singlecell MMSE estimation of the channel $\mathbf{H}_{l l}$ of the $l$ th cell is given by

$$
\widehat{\mathbf{H}}_{l l}=\sqrt{p_{r} \tau} \mathbf{D}_{l l} \mathbf{\Psi}_{l}^{H}\left(\mathbf{I}+p_{r} \tau \boldsymbol{\Psi}_{l} \mathbf{D}_{l l}^{2} \mathbf{\Psi}_{l}^{H}\right)^{-1} \mathbf{Y}_{l}
$$

The estimator of (12) performs well in single-cell scenario. However, it is not capable of eliminating pilot contamination in multicell cases, since the pilot signals from users of neighboring cells are not utilized in the estimation.

3.2. Multicell MMSE Channel Estimation. To mitigate the aforementioned pilot contamination in a multicell scenario, which is always the case in a real network, a straightforward approach is to combine the pilot signals of the users in neighboring cells in the estimator, as in TD-SCDMA system [13].

By reorganizing the pilot matrices $\Psi_{j}$ and channel matrices $\mathbf{G}_{j l},(4)$ can be rewritten as

$$
\begin{aligned}
\mathbf{Y}_{l} & =\sqrt{p_{r} \tau}\left[\begin{array}{lll}
\mathbf{\Psi}_{1} & \ldots & \mathbf{\Psi}_{L}
\end{array}\right]\left[\begin{array}{c}
\mathbf{G}_{1 l} \\
\vdots \\
\mathbf{G}_{L l}
\end{array}\right]+\mathbf{W}_{l} \\
& =\sqrt{p_{r} \tau} \boldsymbol{\Phi} \mathbf{G}_{l}+\mathbf{W}_{l} \\
& =\sqrt{p_{r} \tau} \boldsymbol{\Phi} \mathbf{D}_{l} \mathbf{H}_{l}+\mathbf{W}_{l},
\end{aligned}
$$

where $\Phi \in \mathbb{C}^{\tau \times K L}, \mathbf{G}_{l} \in \mathbb{C}^{K L \times M}, \mathbf{D}_{l}=\left[\begin{array}{ccc}\mathbf{D}_{1 l} & \cdots & 0 \\ \vdots & \ddots & \vdots \\ 0 & \cdots & \mathbf{D}_{L l}\end{array}\right] \epsilon$ $\mathbb{C}^{K L \times K L}, \mathbf{H}_{l}=\left[\begin{array}{c}\mathbf{H}_{1 l} \\ \vdots \\ \mathbf{H}_{L l}\end{array}\right] \in \mathbb{C}^{K L \times M}$.

Then, we have the estimation of the channel response of all the users in all the cells by simply applying the MMSE criterion on (13) [5] as

$$
\begin{array}{r}
\widehat{\mathbf{H}}_{j l}=\sqrt{p_{r} \tau} \mathbf{D}_{j l} \Psi_{j}^{H}\left(\mathbf{I}+p_{r} \tau \sum_{i=1}^{L} \boldsymbol{\Psi}_{i} \mathbf{D}_{i l}^{2} \boldsymbol{\Psi}_{i}^{H}\right)^{-1} \mathbf{Y}_{l}, \\
j=1 \cdots L .
\end{array}
$$

With this, the pilot contamination can be removed jointly. Furthermore, the channel estimation of all the $L$ cells can be described by a unified matrix as

$$
\widehat{\mathbf{H}}_{l}=\sqrt{p_{r} \tau} \mathbf{D}_{l} \boldsymbol{\Phi}^{H}\left(\mathbf{I}+p_{r} \tau \boldsymbol{\Phi} \mathbf{D}_{l}^{2} \boldsymbol{\Phi}^{H}\right)^{-1} \mathbf{Y}_{l} .
$$

However, the abovementioned multicell joint channel estimation works more in theory than in practice, for the following reasons.
Firstly, in the abovementioned channel estimation schemes, either single-cell or multicell, $\left\{\beta_{j l k}\right\}$ are assumed to be fixed, by considering the fact that the large-scale fading (including pathloss and shadow fading) is known to the receivers of all BS and that only the small-scale fading coefficients $\left\{h_{j l k m}\right\}$ need to be estimated $[5,7]$. In a real network scenario, though changing slowly, the large scale fading must be estimated at the receiver and updated from time to time. Furthermore, the estimation error of $\left\{\beta_{j l k}\right\}$ impacts significantly on the performance of uplink data decoding and downlink transmission (e.g., precoding and beamforming).

Secondly, for the large-scale antenna array deployed at BS of a massive MIMO system, the number of antenna elements is in the order of several tens or even hundreds. Thus, the dimension of the matrices in the channel estimation is about one order of magnitude higher than in a normal MIMO system. On the other hand, for the multicell joint channel estimation by (14), the dimension of matrices is even larger, since all the users in the neighboring cells are included in the matrices. Therefore, they are difficult to be applied in practical systems due to the huge computational burden.

In addition, for the joint channel estimation scheme of the target cell $l$ by (14), it can be seen that the knowledge of the large-scale fading from all the cells to the target cell, $\mathbf{D}_{i l}, i=$ $1 \sim L$, is required in advance. Actually it is not necessary by the method as illustrated below.

First, the full channel response $\mathbf{G}_{l l}$ instead of only the small-scale fading $\mathbf{H}_{l l}$ is estimated by MMSE criterion; that is,

$$
\widehat{\mathbf{G}}_{l l}=\sqrt{p_{r} \tau} \boldsymbol{\Psi}_{l}^{H}\left(\mathbf{I}+p_{r} \tau \sum_{i=1}^{L} \boldsymbol{\Psi}_{i} \boldsymbol{\Psi}_{i}^{H}\right)^{-1} \mathbf{Y}_{l}
$$

Then, by left multiplexing both sides of (16) with $\mathbf{D}_{l l}^{-1}$, we have the estimation of the small-scale fading as

$$
\widehat{\mathbf{H}}_{l l}=\sqrt{p_{r} \tau} \mathbf{D}_{l l}^{-1} \boldsymbol{\Psi}_{l}^{H}\left(\mathbf{I}+p_{r} \tau \sum_{i=1}^{L} \boldsymbol{\Psi}_{i} \boldsymbol{\Psi}_{i}^{H}\right)^{-1} \mathbf{Y}_{l}
$$

Compared with (14), obviously the estimation of $\mathbf{D}_{i l}(i \neq l)$ is eliminated in (17). With this, not only the unnecessary computational burden is eliminated, but also the impact of estimation error of $\mathbf{D}_{i l}(i \neq l)$ on the performance of $\mathbf{H}_{l l}$ estimation is mitigated. But, the computational complexity is still huge due to the large number of users and antennas to be considered in the estimation.

3.3. Improved Multicell MMSE Channel Estimation. As we know, the purpose of the joint estimation by including the users of the neighboring cells in the estimation is to mitigate the interference of the pilots in the neighboring cells, so as to improve the performance of channel estimation of users in the target cell. It is not necessary to estimate the channels of the users in the neighboring cells. In view of this, one needs to include only the strong interfering users (SIU) in the neighboring cells into the channel estimator. Then, a compromise between estimation performance and 
computational cost can be achieved, by selecting a proper number of strong interfering users. An improved multicell joint channel estimation is now proposed based on this reasoning.

The basic ideas of the scheme are as follows. Firstly, the large-scale fading is estimated by a matched filter- (MF-) based fast channel estimation. Then, the strong interfering users can be decided by sorting the pilot signal strength. Also, the estimation of large-scale fading can be utilized in the joint channel estimation thereafter. Considering the poor performance of MF in non-Gaussian scenario, signal reconstruction and interference cancellation are employed to improve the estimation performance of the large-scale fading. On the basis of this, both the users of target cell and the strong interfering users of the neighboring cells are included in the channel and the system matrices of the multicell joint MMSE estimation. Then, a precise estimation of the smallscale fading of the target cell users can be achieved. The block diagram of the proposed scheme is illustrated in Figure 2.

In view of the outstanding autocorrelation and crosscorrelation properties of the Chu-based pilot sequence and its promising capability in the reduction of the nonorthogonality of intercell users, here we employ the pilot design given in [10]. Details of the proposed scheme are as follows.

(1) MF-Based Estimation of Large-Scale Fading of Target Cell. Taking the second item of (5) as interference, the initial channel estimation of the $l$ th cell is obtained by employing the MF theorem as in [14]:

$$
\widehat{\mathbf{G}}_{l l}^{\mathrm{MF}}=\frac{1}{\sqrt{p_{r} \tau}} \operatorname{diag}\left(\boldsymbol{\Psi}_{l}^{H} \boldsymbol{\Psi}_{l}\right)^{-1} \boldsymbol{\Psi}_{l}^{H} \mathbf{Y}_{l}
$$

Note that $\Psi$ of all the cells are known a priori; therefore, $\operatorname{diag}\left(\boldsymbol{\Psi}_{l}^{H} \boldsymbol{\Psi}_{l}\right)^{-1} \boldsymbol{\Psi}_{l}^{H}$ can be calculated and stored in advance.

Since the large-scale fading of one user to all the antennas of one BS is almost the same, and that, in the case of $M \gg$ $K$, the fast fading is assumed to be i.i.d. and $\mathscr{C} \mathscr{N}(0,1)$, the large scale fading of each of the users in the target cell can be calculated as

$$
\sqrt{\sqrt{\beta_{l l k}}}=\frac{1}{Q} \mathbb{E}\left[\widehat{\mathbf{G}}_{l l}^{\mathrm{MF}}\right]_{k}, \quad k=1, \ldots, K .
$$

Here $Q$ is the normalization factor for fast fading, which is 1.2535 for $\mathscr{C} \mathscr{N}(0,1)$.

$$
\text { Let } \widehat{\mathbf{D}}_{l l}=\operatorname{diag}\left(\widehat{\sqrt{\beta_{l l 1}}}, \widehat{\sqrt{\beta_{l l 2}}}, \ldots, \widehat{\sqrt{\beta_{l l K}}}\right) \text {. }
$$

(2) Interference Cancellation-Based Large-Scale Fading Estimation of Neighboring Cell Users. Suppose that there are $C_{N}$ neighboring cells around the target cell $l$. Since the pilot strength of the target cell is always the largest in the received pilots, one needs to remove it first so as to improve the precision of the neighboring cell estimation; that is,

$$
\widehat{\mathbf{Y}}_{l}=\mathbf{Y}_{l}-\sqrt{p_{r} \tau} \Psi_{l} \widehat{\mathbf{G}}_{l l}^{\mathrm{MF}} .
$$

By supposing $\widehat{\mathrm{Y}}_{l}$ as the pilots of all the users of the $i$ th cell arriving at the antenna of the $l$ th BS, we have

$$
\widehat{\mathbf{Y}}_{l}=\sqrt{p_{r} \tau} \Psi_{i} \mathbf{G}_{i l}+\mathbf{W}_{l} .
$$

Then, the large-scale fading of the links between the users of the $i$ th cell and the antenna of the $l$ th BS can be estimated using (18) and (19).

All the $C_{N}$ neighboring cells can be processed sequentially in the same way as in (18) and (19), by replacing the system matrix $\Psi_{i}$ with the pilot sequences of the corresponding neighboring cell. With this, the large-scale fading of all the users in all the $C_{N}$ neighboring cells are obtained.

(3) Selection of Strong Interfering Users. Sort all the neighboring cell users, in an ascending order of its large-scale fading. Taking the top $K_{1}$ users in the cell list as the "strong interfering users," we define the large-scale fading matrix as $\widehat{\mathbf{D}}_{l}^{\mathrm{CN}}=\operatorname{diag}\left(\widehat{\sqrt{\beta_{n l 1}}}, \widehat{\sqrt{\beta_{n l 2}}}, \ldots, \sqrt{\sqrt{\beta_{n l K_{1}}}}\right)$, where $n$ denotes the cell which the $k$ th SIU belongs to. The value of $K_{1}$ is decided by the remaining capability for the processing of users other than the target cell users. Suppose that the overall capability of the BS is to estimate the channel response of $K_{a}$ users and that the number of active users of the target cell is $K_{0}\left(K_{0} \leqslant K\right)$; then $K_{1}=K_{a}-K_{0}$ is the number of SIUs to be processed.

It should be noted that, since the large-scale fading always changes slowly, Steps (1) (3) need not be carried out after every frame, but carried out, say, after every ten frames, so as to save unnecessary computational burden.

(4) Generation of the System Matrix for Joint Channel Estimation. Putting together the estimated large-scale fading of all the $K_{0}$ users in the target cell and the $K_{1}$ SIUs of the neighboring cells, we have a new large-scale fading matrix given by

$$
\widehat{\mathbf{D}}_{l}=\left[\begin{array}{cc}
\widehat{\mathbf{D}}_{l}^{\mathrm{CT}} & 0 \\
0 & \widehat{\mathbf{D}}_{l}^{\mathrm{CN}}
\end{array}\right] \in \mathbb{R}^{K_{a} \times K_{a}},
$$

where $\widehat{\mathbf{D}}_{l}^{\mathrm{CT}}=\operatorname{diag}\left(\widehat{\sqrt{\beta_{l l 1}}}, \widehat{\sqrt{\beta_{l l 2}}}, \ldots, \sqrt{\sqrt{\beta_{l l K_{0}}}}\right)$. The system matrix can then be defined accordingly, by combining the pilot sequence of the $K_{0}$ users of the target cell and the $K_{1}$ strong interfering users of neighboring cells, as

$$
\underline{\Psi}_{l}=\left[\begin{array}{lllll}
\psi_{l 1} & \cdots & \underline{\psi}_{l K_{0}} \underline{\psi}_{n 1} & \cdots & \underline{\psi}_{n K_{1}}
\end{array}\right] \in \mathbb{C}^{\tau \times K_{a}} .
$$

Denoting $\underline{\mathbf{H}}_{l} \in \mathbb{C}^{K_{a} \times M}$ as the small-scale fading of the $K_{0}$ users of target cell and the $K_{1}$ SIUs of neighbor cells, the received pilot signal by the target cell is then given by

$$
\mathbf{Y}_{l}=\sqrt{p_{r} \tau} \underline{\Psi}_{l} \underline{\mathbf{D}}_{l} \underline{\mathbf{H}}_{l}+\underline{\mathbf{W}}_{l},
$$

where $\mathbf{W}_{l}$ refers to the noise and received pilot signal of all the users excluding the abovementioned $K_{0}+K_{1}$ users.

(5) MMSE-Based Joint Channel Estimation. By applying the MMSE criterion to (24), we have the estimation of the smallscale fading of target cell users along with the SIUs as

$$
\underline{\widehat{\mathbf{H}}}_{l}=\sqrt{p_{r} \tau} \underline{\mathbf{D}}_{l} \underline{\Psi}_{l}^{H}\left(\mathbf{I}+p_{r} \tau \underline{\Psi}_{l} \underline{\mathbf{D}}_{l}^{2} \underline{\Psi}_{l}^{H}\right)^{-1} \mathbf{Y}_{l}
$$

in which $\underline{\widehat{\mathbf{H}}}_{l} \in \mathbb{C}^{K_{a} \times M}$. Taking the first $K_{0}$ rows of $\underline{\mathbf{H}}_{l}$, we then have the final estimation of small scale fading of the target cell. 


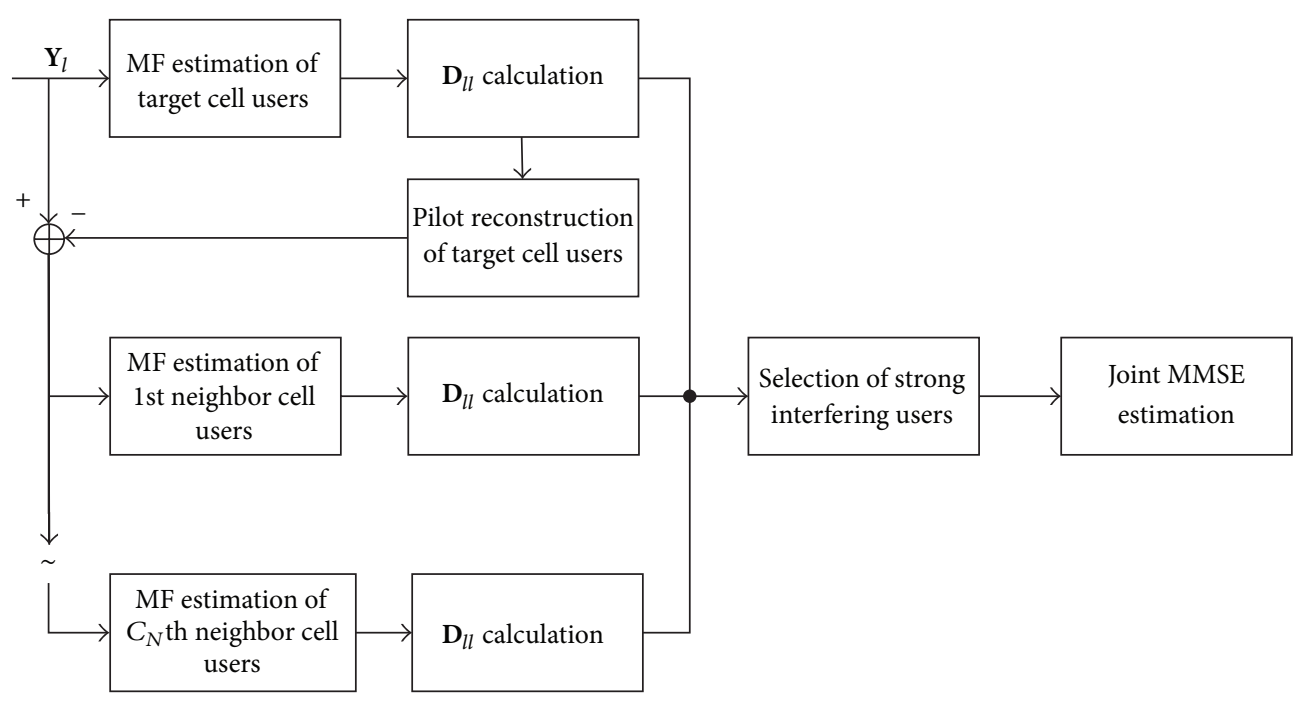

FIGURE 2: Block diagram for an improved joint MMSE channel estimation.

\section{Experimental Results}

4.1. Assumptions of Simulation. In order to evaluate the proposed scheme, simulations of a massive MIMO system were performed. The system consists of 7 cells sharing the same band of frequencies, with 6 cells surrounding one cell and the cell edges of the 6 cells overlapping the edges of the central cell. The parameters of the system are given in Table 1.

The terminals are distributed uniformly in the assigned cells (no handover across the cells). The fast fading factor of every uplink terminal-BS path is generated randomly following i.i.d. $\mathscr{C} \mathscr{N}(0,1)$ and updated every frame. The distances between every terminal-BS pair are updated every 10 frames. The shadow fading is modeled with log-normal distribution and is the same between each user and all BSs. More explicitly, the large-scale fading coefficient between each user and BS can be expressed as $\sqrt{\beta_{l l k}}=10^{w / 10} /\left(r_{j l k} / 100\right)^{v}$ when the power is measured in Watts, where $r_{j l k}$ is the distance between the $k$ th terminal in the $j$ th cell to BS in the $l$ th cell, $w$ is the corresponding shadow fading coefficient in $\mathrm{dB}$, and denoted as a Gaussian random variable with zero mean and variance $\sigma_{s}^{2}\left(\right.$ i.e., $\left.\mathcal{N}\left(0, \sigma_{s}^{2}\right)\right)$.

The simulations are performed in an AWGN noisy environment, with the transmit power for both the pilots and the data being the same for each terminal and controlled by a predefined Tx power to noise ratio.

For the pilots used in the simulation, the same setting as in [10] is employed. That is, the sequence parameter $N$ in (7) is set to be unity for all the seven cells, and the same sequence is cyclic shifted for terminals in the same cell. And the parameters $q_{i}, i=1,2, \ldots, 7$ in (6) are set to be $\{0,1,2,9,10,11,12\}$ for the seven cells. Furthermore, to mitigate the impact of the correlation property of the pilots onto the performance of channel estimation, the pilot sequences with less cross correlation are assigned to the strong interfering users in the simulation.
TABLE 1: Simulation parameters.

\begin{tabular}{lc}
\hline Parameter & Value \\
\hline Cell radius $(R)$ & $500 \mathrm{~m}$ \\
Pathloss exponent $(v)$ & 3.8 \\
Carrier frequency $\left(f_{c}\right)$ & $2 \mathrm{GHz}$ \\
Shadow fading standard deviation $\left(\sigma_{s}\right)$ & $8 \mathrm{~dB}$ \\
Number of subcarriers for pilots $\left(N_{s}\right)$ & 12 \\
Number of symbols for pilots $\left(N_{c}\right)$ & 3 \\
Pilot length $(\tau)$ & 36 \\
Number of cells $(L)$ & 7 \\
Number of users per cell $(K)$ & 24 \\
Number of strong interfering users in neighbor & 12 \\
cells $\left(K_{1}\right)$ & 100 \\
Number of BS antennas $(M)$ & $10 \mathrm{~ms}$ \\
Frame length
\end{tabular}

4.2. Metrics Used for the Evaluation of Channel Estimation Error. To evaluate the proposed channel estimation scheme, three performance metrics are defined here, one for the evaluation of large-scale fading and the other two for the small-scale fading.

The first one is a mean square error (MSE) of the largescale fading estimation for all the paths between all the $K \times L$ users in the network and the $l$ th BS given by

$$
\omega \triangleq \mathbb{E}\left(\left|\sqrt{\sqrt{\beta_{l k}}}-\sqrt{\beta_{l k}}\right|^{2}\right), \quad k=1, \ldots, K \times L .
$$

This metric gives an evaluation of the estimation error of large-scale fading. 
TABLE 2: Impact of large-scale fading estimation and SIU selection on the performance of small-scale fading estimation.

\begin{tabular}{llr}
\hline Scheme & $\varepsilon(\mathrm{dB})$ & $\rho$ \\
\hline Case 1: proposed scheme with ideal $\beta$ & -8.91 & 0.933 \\
Case 2: proposed scheme with estimated $\beta$ & -7.53 & 0.911 \\
Case 3: proposed scheme with ideal $\beta$ but randomly selected SIUs & -2.29 & 0.806 \\
\hline
\end{tabular}

The second one is a normalized channel estimation error $(\varepsilon)$ of small-scale fading given by

$$
\varepsilon \triangleq 10 \log _{10}\left(\sum_{k=1}^{K} \sum_{m=1}^{M} \frac{\left\|\left[\widehat{\mathbf{H}}_{l l}\right]_{k, m}-\left[\mathbf{H}_{l l}\right]_{k, m}\right\|^{2}}{\left\|\left[\mathbf{H}_{l l}\right]_{k, m}\right\|^{2}}\right),
$$

where $\mathbf{H}_{l l}$ and $\widehat{\mathbf{H}}_{l l}$ are the desired small-scale channel response at the $l$ th base station and its estimate, respectively. Although the channel response of the top $K_{1}$ strongest interfering users in the neighboring cells has also been estimated by the proposed multicell channel estimation scheme, the channel estimation errors of these users are not of interest to us. Therefore, here we consider only the channel estimation error of the desired channel of the $l$ th cell. The smaller the value of $\varepsilon$, the better the channel estimation.

The third metric is the channel correlation coefficient as defined in (28), which gives an evaluation of the normalized correlation property between the channel estimation and the desired channel:

$$
\rho \triangleq\left|\frac{\operatorname{cov}\left(\widehat{\mathbf{H}}_{l l}, \mathbf{H}_{l l}\right)}{\sqrt{\operatorname{cov}\left(\widehat{\mathbf{H}}_{l l}, \widehat{\mathbf{H}}_{l l}\right) \operatorname{cov}\left(\mathbf{H}_{l l}, \mathbf{H}_{l l}\right)}}\right|,
$$

where $\operatorname{cov}(\mathbf{a}, \mathbf{b})$ refers to the covariance between $\mathbf{a}$ and $\mathbf{b}$. Generally, the correlation coefficient approaches unity as the performance of the channel estimation improves. Just as in the definition of the channel estimation error, only the channel of the target cell is considered for channel correlation coefficient.

4.3. Simulation Results. By setting different levels of noise and applying the proposed channel estimation scheme, we obtain MSE of the estimation of large-scale fading, as illustrated in Figure 3. It can be seen that the estimation error is marginal even in the case of rather strong background noise being present.

To evaluate the impact of the estimation error of the largescale fading on the small-scale fading estimation, we compare the normalized channel estimation errors and the channel correlation coefficients under three schemes: the proposed scheme, the proposed scheme with an ideal knowledge of the large-scale fading, and the proposed scheme with an ideal knowledge of the large-scale fading but with randomly selected SIUs. The simulation results are given in Table 2, which shows that the estimation error of the large-scale fading has a significant impact on the performance of the small-scale fading estimation. This is mainly due to a wrong selection of SIUs with poor estimation of the large-scale fading. By comparing Case 1 and Case 3, it is obvious that,

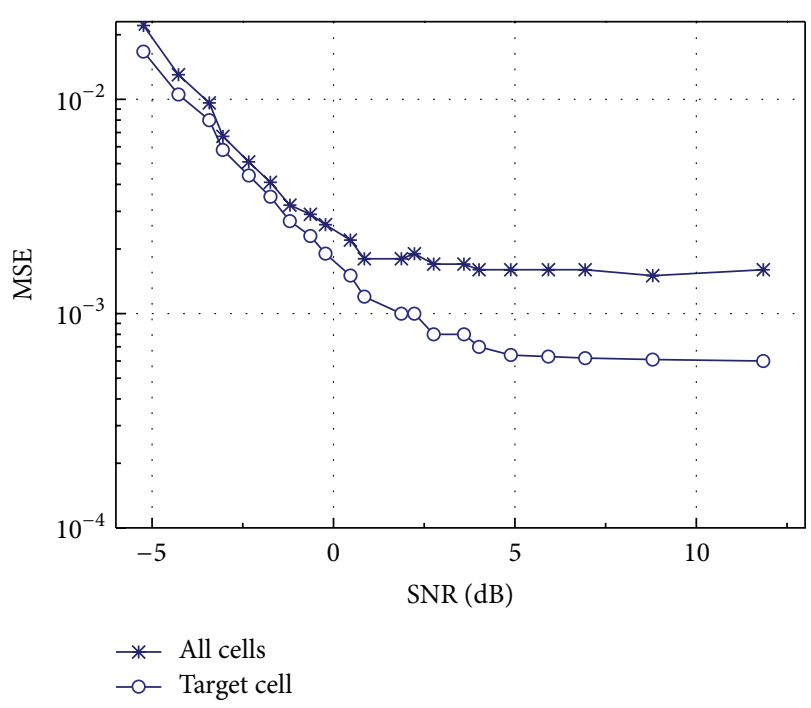

FIGURE 3: Performance of large-scale fading estimation versus channel condition.

TABLE 3: Performance comparison of channel estimation schemes.

\begin{tabular}{lcc}
\hline Channel estimation scheme & $\varepsilon(\mathrm{dB})$ & $\rho$ \\
\hline Single-cell MMSE $\left(K_{1}=0\right)$ & -2.36 & 0.828 \\
Improved multicell MMSE $\left(K_{1}=4\right)$ & -5.33 & 0.879 \\
Improved multicell MMSE $\left(K_{1}=12\right)$ & -6.72 & 0.901 \\
Improved multicell MMSE $\left(K_{1}=24\right)$ & -8.41 & 0.927 \\
Improved multicell MMSE $\left(K_{1}=48\right)$ & -9.23 & 0.938 \\
Improved multicell MMSE $\left(K_{1}=96\right)$ & -8.96 & 0.934 \\
Full-cell MMSE $\left(K_{1}=144\right)$ & -8.86 & 0.933 \\
\hline
\end{tabular}

even with an ideal estimation of the large-scale fading, a wrong selection of SIUs impacts severely on the estimation of the small-scale fading.

To further evaluate the proposed scheme, we now compare the performance of channel estimation in the case of conventional schemes and the proposed scheme, all based on the estimated large-scale fading by the proposed interference cancellation-based algorithm. The results are listed in Table 3. Two conventional schemes are considered here. One is the single-cell MMSE where no neighboring cell users are included in the channel estimation, and the other is the multicell MMSE channel estimation with all the neighboring cell users included in the joint processing (referred as full-cell MMSE channel estimation). The proposed scheme with different number of SIUs is compared 
TABLE 4: Comparison of computational cost (unit: MIPS).

\begin{tabular}{|c|c|c|c|c|}
\hline Channel estimation scheme & Large-scale fading estimation & Small-scale fading estimation & Total & Total savings \\
\hline Improved multicell MMSE $\left(K_{1}=12\right)$ & 28 & 136.9 & 164.9 & - \\
\hline Full-cell MMSE & 28 & 397.3 & 425.3 & $61.2 \%$ \\
\hline Single-cell MMSE & 28 & 113.2 & 141.2 & $-16.8 \%$ \\
\hline
\end{tabular}

as well, so as to find a reasonable number of neighboring users in the joint estimation. From Table 3 it can be seen that the proposed scheme performs much better than the singlecell MMSE scheme does and approaches that of the ideal multicell MMSE with a slight degradation when the number of SIUs considered in the joint processing is not less than 24, which is equal to the total number of users in a cell. When the number of SIUs is larger than 48, the estimation performance is almost the same as that of a full-cell MMSE.

4.4. Computational Complexity. The computational complexity of the proposed algorithm is now evaluated and compared with that of the conventional ones. Suppose that one instruction cycle is needed for each real multiply-add operation in the digital signal processor (DSP).

For the proposed algorithm, since $\operatorname{diag}\left(\boldsymbol{\Psi}_{l}^{H} \boldsymbol{\Psi}_{l}\right)^{-1} \boldsymbol{\Psi}_{l}^{H}$ in (18) of Step (1) can be computed and stored in advance, there is no real time computation consumed for this part. The computation in (18) costs $K \times \tau \times M$ complex multiply-add operations. In (19), additional $K \times M$ complex add operations are needed. In total 350,400 real operations are needed per iteration. For Step (2), the pilot reconstruction of the target cell and interference deduction in (20) cost $K \times \tau \times M$ complex multiply-add and $\tau \times M$ complex subtract operations. Along with the MF estimation and large-scale fading computation as in (18) and (19) for each neighboring cell, the overall computational cost in Step (2) is 2,455,200 real operations per iteration. The computational burden of Steps (3) and (4) is negligible. For Step (5), the overall computational cost in (25) consists of $K_{a} \times \tau$ complex multiply, $K_{a}$ real multiply, $K_{a} \times \tau$ complex multiply, $\tau \times K_{a} \times \tau$ complex multiply-add, $\tau$ real add, $\tau \times \tau \times M$ complex multiply-add, $\tau \times \tau$ complex matrix inversion, and $K_{a} \times \tau \times M$ complex multiply-add operations, which amount to a total of $1,368,648$ real operations for one frame.

Assume Steps (1) and (2) are computed every 10 frames, and Step (5) is computed each frame; then the computation cost for the estimation of the large-scale fading (i.e., Steps (1) and (2)) and the small-scale fading are 28 MIPS and 136.9 MIPS, respectively. Thus, the total computation cost for the proposed algorithm is 164.9 MIPS.

The computational cost for the full-cell MMSE estimation and the single-cell MMSE estimation may be obtained in a similar manner and the results are compared with those of the proposed algorithm in Table 4 . From this table, it can be seen that the proposed algorithm saves $61.2 \%$ computational cost with respect to that of the full-cell MMSE scheme, and the additional computational burden over that of the singlecell MMSE is $16.8 \%$, which is rather small.

\section{Conclusions}

In this paper, the pilot contamination problem of a massive MIMO system is discussed. In view of the fact that currently the channel estimation schemes for massive MIMO systems always assume that the large-scale fading is known a priori and that the computational complexity of conventional channel estimations grows sharply in massive MIMO systems, an improved multicell MMSE joint channel estimation scheme, which aims to mitigate the pilot contamination at an affordable expense and in a more realistic situation, has been proposed.

In the proposed scheme, the highly interfering users in neighboring cells have been identified first based on the estimation of the large-scale fading and then included in the joint channel processing. Metrics of the performance evaluation of the channel estimation have been defined. Finally, the simulation results and the computational complexity analysis are performed that verify the performance advantage of the proposed scheme at a reasonable computational cost over that of the conventional schemes.

\section{Conflict of Interests}

The authors declare that there is no conflict of interests regarding the publication of this paper.

\section{Acknowledgment}

This work was supported by the National Natural Science Foundation of China under Grant no. 61301103.

\section{References}

[1] T. L. Marzetta, "Noncooperative cellular wireless with unlimited numbers of base station antennas," IEEE Transactions on Wireless Communications, vol. 9, no. 11, pp. 3590-3600, 2010.

[2] E. G. Larsson, F. Tufvesson, O. Edfors, and T. L. Marzetta, "Massive MIMO for next generation wireless systems," IEEE Communications Magazine, vol. 52, no. 2, pp. 186-195, 2014.

[3] H. Q. Ngo, E. Larsson, and T. Marzetta, "Energy and spectral efficiency of very large multiuser MIMO systems," IEEE Transactions on Communications, vol. 61, no. 4, pp. 1436-1449, 2013.

[4] H. Ngo, E. Larsson, and T. Marzetta, “The multicell multiuser mimo uplink with very large antenna arrays and a finitedimensional channel," IEEE Transactions on Communications, vol. 61, no. 6, pp. 2350-2361, 2013.

[5] J. Jose, A. Ashikhmin, T. L. Marzetta, and S. Vishwanath, "Pilot contamination problem in multi-cell TDD systems," in Proceedings of the IEEE International Symposium on Information 
Theory (ISIT '09), pp. 2184-2188, Seoul, Republic of Korea, July 2009.

[6] J. Jose, A. Ashikhmin, T. L. Marzetta, and S. Vishwanath, "Pilot contamination and precoding in multi-cell TDD systems," IEEE Transactions on Wireless Communications, vol. 10, no. 8, pp. 2640-2651, 2011.

[7] H. Q. Ngo and E. G. Larsson, "EVD-based channel estimations for multicell multiuser MIMO with very large antenna arrays," in Proceedings of the IEEE International Conference on Acoustics, Speech and Signal Processing (ICASSP '12), pp. 3249-3252, 2012.

[8] H. Yin, D. Gesbert, M. Filippou, and Y. Liu, "A coordinated approach to channel estimation in large-scale multiple-antenna systems," IEEE Journal on Selected Areas in Communications, vol. 31, no. 2, pp. 264-273, 2013.

[9] H. Yin, D. Gesbert, M. C. Filippou, and Y. Liu, "Decontaminating pilots in massive MIMO systems," in Proceedings of the IEEE International Conference on Communications (ICC '2013), pp. 3170-3175, 2013.

[10] A. Hu, T. Lv, H. Gao, Y. Lu, and E. Liu, "Pilot design for largescale multi-cell multiuser MIMO systems," in Proceedings of the IEEE International Conference on Communications (ICC '2013), pp. 5381-5385, 2013.

[11] A. Ashikhmin and T. Marzetta, "Pilot contamination precoding in multi-cell large scale antenna systems," in Proceedings of the IEEE International Symposium on Information Theory (ISIT '2012), pp. 1137-1141, 2012.

[12] D. C. Chu, "Polyphase codes with good periodic correlation properties," IEEE Transactions on Information Theory, vol. 18, no. 4, pp. 531-532, 1972.

[13] X. Song, K. Li, and A. Hu, "Two-stage channel estimation algorithm for TD-SCDMA system employing multi-cell joint detection," Circuits, Systems, and Signal Processing, vol. 26, no. 6, pp. 961-968, 2007.

[14] J. G. Proakis, Digital Communications, McGraw-Hill, New York, NY, USA, 4th edition, 2001. 

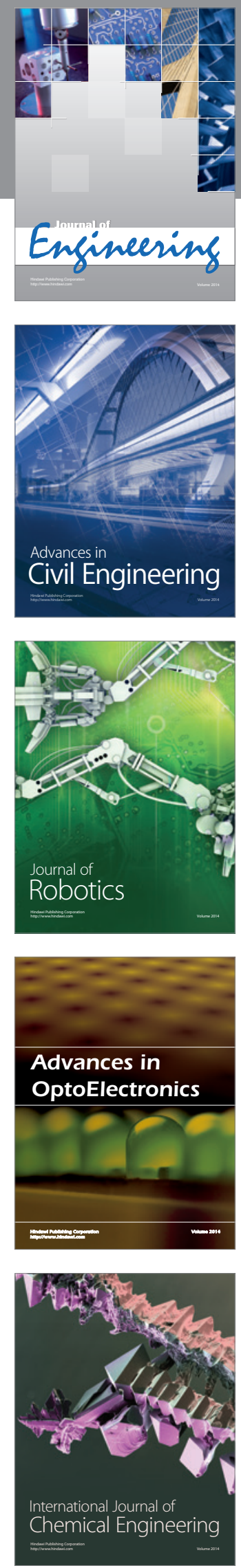

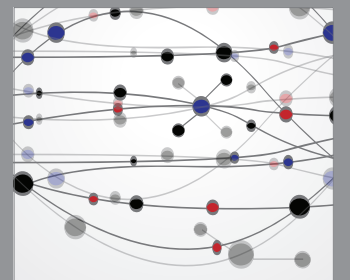

The Scientific World Journal
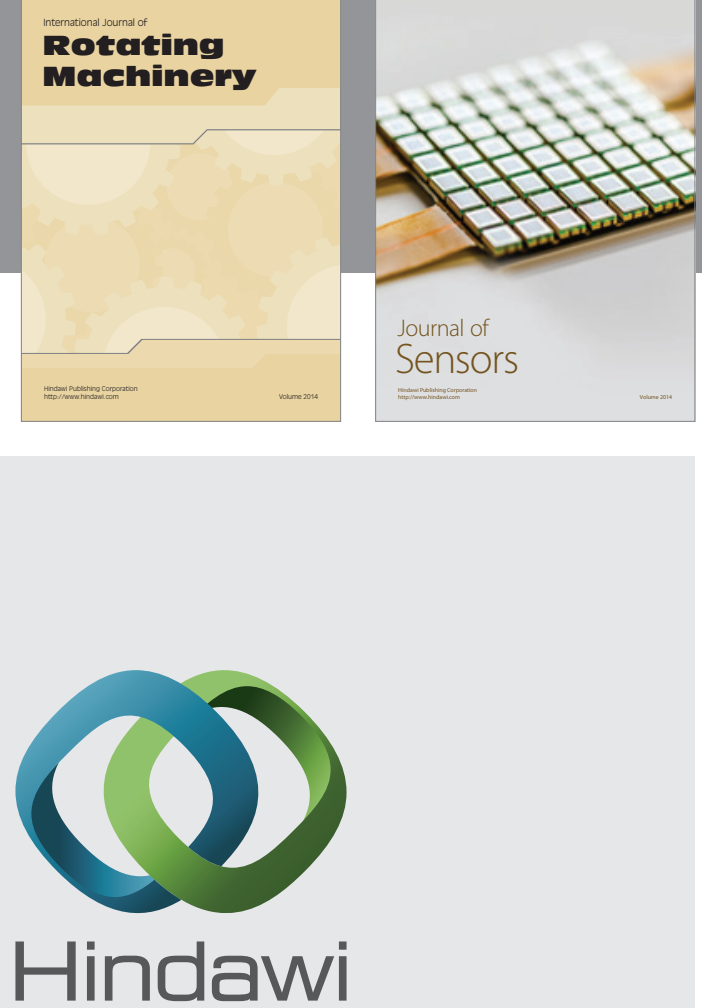

Submit your manuscripts at http://www.hindawi.com
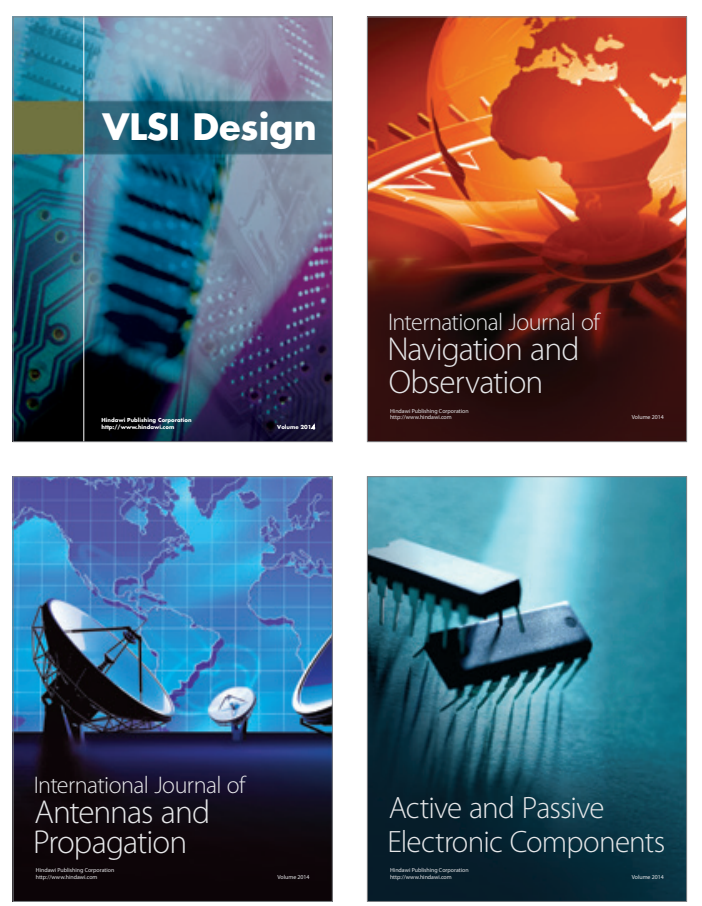
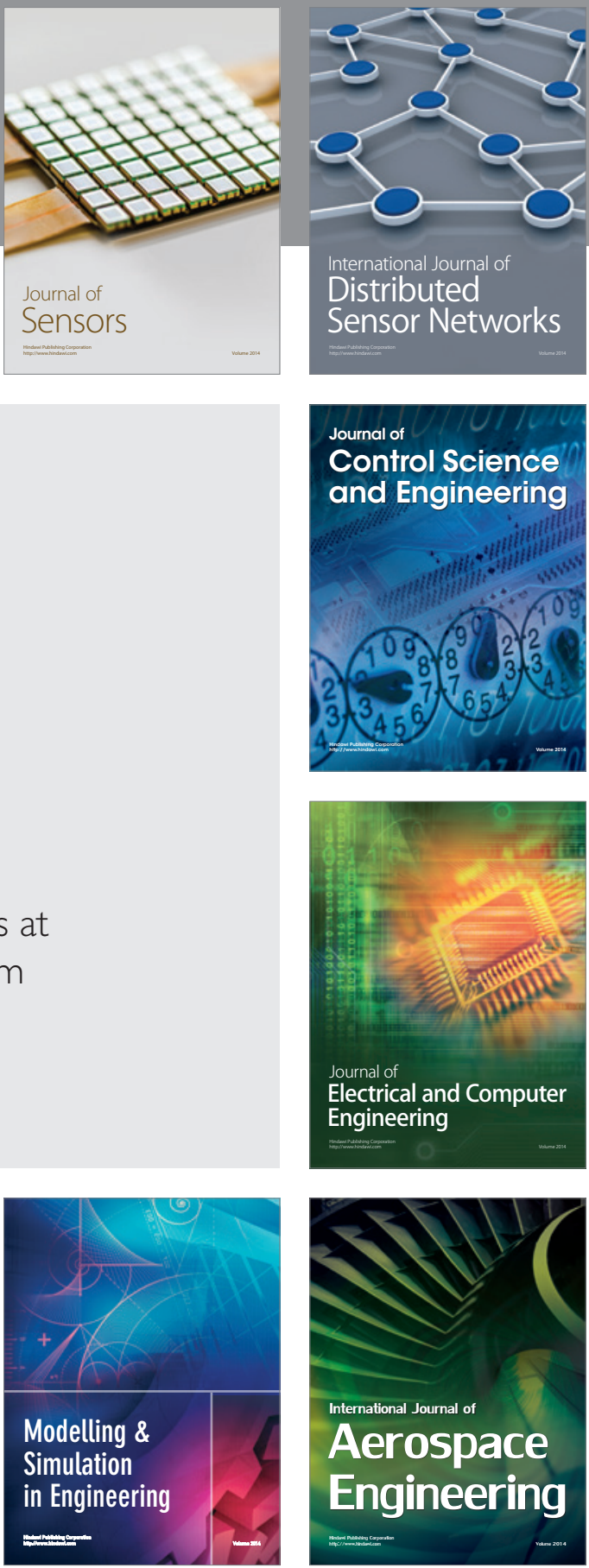

Journal of

Control Science

and Engineering
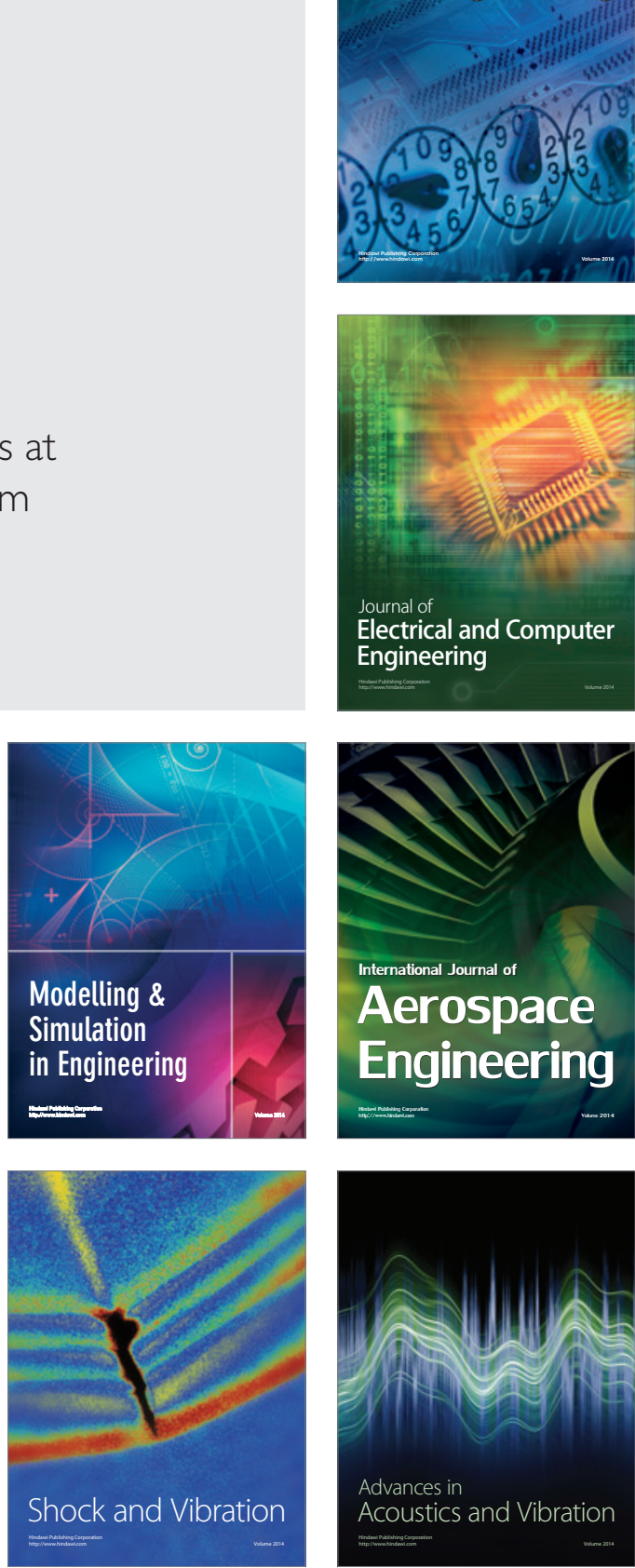